\title{
High voltage asymmetric supercapacitors developed by engineering electrode work functions
}

\author{
Kowsik Sambath Kumar ${ }^{\mathrm{a}, \mathrm{b}}$, Deepak Pandey ${ }^{\mathrm{a}, \mathrm{b}}$ Jayan Thomas* a,b,c
}

${ }^{a}$ Department of Materials Science and Engineering, University of Central Florida, Orlando, Florida 32816, United States.

${ }^{b}$ NanoScience Technology Center, University of Central Florida, Orlando, Florida 32826, United States

${ }^{c}$ CREOL, College of Optics and Photonics, University of Central Florida, Orlando, Florida 32816, United States

Corresponding Author

* Email: Jayan.Thomas@ucf.edu

\section{Experimental Section}

\subsection{Materials}

Carbon fibers (CF) were purchased from Fibre Glast, USA. Nitric Acid $\left(\mathrm{HNO}_{3}\right)$, Hydrochloric acid $(\mathrm{HCl})$, Manganese acetate tetrahydrate $\left[\left(\mathrm{CH}_{3} \mathrm{COO}\right)_{2} \mathrm{Mn} .4 \mathrm{H}_{2} \mathrm{O}\right]$, Sodium sulfate $\left(\mathrm{Na}_{2} \mathrm{SO}_{4}\right)$ Lithium sulfate $\left(\mathrm{Li}_{2} \mathrm{SO}_{4}\right)$, Potassium sulfate $\left(\mathrm{K}_{2} \mathrm{SO}_{4}\right)$, Sodium Molybdate $\left(\mathrm{Na}_{2} \mathrm{MoO}_{4}\right)$, Disodium ethylenediaminetetraacetate dihydrate $\left(\mathrm{Na}_{2}\right.$ EDTA), Ammonium acetate $\left(\mathrm{CH}_{3} \mathrm{COONH}_{4}\right)$ were purchased from Sigma-Aldrich, USA.

\subsection{Cleaning of CF}

Prior to the active material's electrodeposition, each CF bare electrode was cut into $2 \mathrm{~cm} \mathrm{x}$ $4 \mathrm{~cm}$ area and cleaned using $\mathrm{HNO}_{3}, \mathrm{HCl}$ and de-ionized water. It was then dried in an oven for 1 hour.

\subsection{Synthesis of $\mathrm{MnO}_{2}$}

The electrodeposition bath for the synthesis of $\mathrm{MnO}_{2}$ electrode consisted of $0.16 \mathrm{M}$ manganese acetate tetrahydrate and $0.16 \mathrm{M}$ sodium sulfate in de-ionized water. A three-electrode cell configuration was used for depositing $\mathrm{MnO}_{2}$ on $\mathrm{CF}$ substrate in which the CF substrate, platinum foil, and $\mathrm{Ag} / \mathrm{AgCl}$ were used as the working electrode, the counter electrode, and the 
reference electrode, respectively. The electrodeposition technique was done at a constant current of $0.5 \mathrm{~mA} / \mathrm{cm}^{2}$ for a period of 20 minutes, followed by drying in oven at $85{ }^{\circ} \mathrm{C}$.

\subsection{Synthesis of $\mathrm{NaMnO}_{2}$}

Initially, $\mathrm{Mn}_{3} \mathrm{O}_{4}$ nanowalls (NWs) were synthesized on $\mathrm{CF}$ by electrochemical deposition method following our previous work. ${ }^{1}$ The electrodeposition bath consisted of a solution of 0.16 $\mathrm{M}$ manganese acetate tetrahydrate and $0.16 \mathrm{M}$ sodium sulfate in de-ionized water. A threeelectrode cell configuration was used for depositing $\mathrm{Mn}_{3} \mathrm{O}_{4} \mathrm{NWs}$ on CF substrate in which the CF substrate, platinum foil, and $\mathrm{Ag} / \mathrm{AgCl}$ were used as the working electrode, the counter electrode, and the reference electrode, respectively. The electrodeposition technique was done at a constant voltage of $-1.8 \mathrm{~V}$ for a period of 20 minutes, followed by drying at room temperature overnight to form $\mathrm{Mn}_{3} \mathrm{O}_{4} \mathrm{NWs}$. This $\mathrm{Mn}_{3} \mathrm{O}_{4} / \mathrm{CF}$ was electrochemically oxidized in a $1 \mathrm{M} \mathrm{Na}_{2} \mathrm{SO}_{4}$ solution to form $\mathrm{NaMnO}_{2}$ by cycling them from 0 to $1.3 \mathrm{~V}$ at $25 \mathrm{mV} \mathrm{s}^{-1}$ for 500 cycles. The obtained sample was cleaned in DI water and dried in oven before weighing and testing them. The mass loading was found to be $\sim 3 \mathrm{mg} \mathrm{cm}^{-2}$.

\subsection{Synthesis of $\mathrm{LiMnO}_{2}$}

An electrodeposition bath consisting of a $0.16 \mathrm{M}$ manganese acetate tetrahydrate solution, and a $0.16 \mathrm{M}$ lithium sulfate solution was used for the synthesis. A three-electrode cell configuration was used for depositing $\mathrm{Mn}_{3} \mathrm{O}_{4} \mathrm{NWs}$ on $\mathrm{CF}$ substrate. The CF substrate, platinum foil, and $\mathrm{Ag} / \mathrm{AgCl}$ were used as the working electrode, the counter electrode, and the reference electrode, respectively. The electrodeposition technique was done at a constant voltage of $-1.8 \mathrm{~V}$ for a period of 20 minutes, followed by drying at room temperature overnight to form $\mathrm{Mn}_{3} \mathrm{O}_{4} \mathrm{NWs}$. These $\mathrm{Mn}_{3} \mathrm{O}_{4} / \mathrm{CF}$ were electrochemically oxidized in a $1 \mathrm{M} \mathrm{Li}_{2} \mathrm{SO}_{4}$ solution to form $\mathrm{LiMnO}_{2}$ by cycling them from 0 to $1.3 \mathrm{~V}$ at $25 \mathrm{mV} \mathrm{s}^{-1}$ for 500 cycles. The obtained sample was cleaned in DI water and dried in oven before weighing and testing them. The mass loading was found to be $\sim 3$ $\mathrm{mg} \mathrm{cm} \mathrm{cm}^{-2}$.

\subsection{Synthesis of $\mathrm{KMnO}_{2}$}

The electrodeposition bath contained a solution of $0.16 \mathrm{M}$ manganese acetate tetrahydrate and $0.16 \mathrm{M}$ potassium sulfate in de-ionized water. A three-electrode cell configuration was used to deposit $\mathrm{Mn}_{3} \mathrm{O}_{4} \mathrm{NWs}$ on the CF substrate. The CF substrate, platinum foil, and $\mathrm{Ag} / \mathrm{AgCl}$ were used as the working electrode, the counter electrode, and the reference electrode, respectively. The electrodeposition technique was done at a constant voltage of $-1.8 \mathrm{~V}$ for a period of 20 minutes, followed by drying at room temperature overnight to form $\mathrm{Mn}_{3} \mathrm{O}_{4} \mathrm{NWs}$. These $\mathrm{Mn}_{3} \mathrm{O}_{4} / \mathrm{CF}$ were electrochemically oxidized in a $1 \mathrm{M} \mathrm{K}_{2} \mathrm{SO}_{4}$ solution to form $\mathrm{KMnO}_{2}$ by cycling them from 0 to $1.3 \mathrm{~V}$ at $25 \mathrm{mVs}^{-1}$ for 500 cycles. The obtained sample was cleaned in DI water and dried in oven before weighing and testing them. The mass loading was found to be $\sim 3 \mathrm{mg} \mathrm{cm}^{-2}$.

\subsection{Synthesis of $\mathrm{MoO}_{2}$}

Molybdenum oxide was also electrodeposited on CF. A solution of $0.10 \mathrm{M}$ sodium molybdate, $0.10 \mathrm{M} \mathrm{Na}_{2}$ EDTA and $0.10 \mathrm{M}$ ammonium acetate was used as the electrolyte in the 
bath. The $\mathrm{CF}$ substrate, platinum foil, and $\mathrm{Ag} / \mathrm{AgCl}$ were used as the working electrode, the counter electrode, and the reference electrode, respectively. The molybdenum oxide film was formed at a constant voltage of $-2 \mathrm{~V}$ applied at the working electrode under $70^{\circ} \mathrm{C}$ for 30 mins. After the deposition, the electrode was washed with de-ionized water and annealed at $400{ }^{\circ} \mathrm{C}$ for 1 hour in the Nitrogen atmosphere. The mass loading was found to be $\sim 4 \mathrm{mg} \mathrm{cm}^{-2}$.

\subsection{Material characterization}

The surface morphology of the electrodeposited active materials on CF was characterized by SEM (Zeiss ULTRA-55 FEG SEM). The SEM (Zeiss ULTRA-55 FEG SEM) is equipped with the Noran System 7 EDS system with Silicon Drift Detector to perform the energy-dispersive Xray spectroscopy. The structure of the materials was determined using XRD analysis (PANalytical Empyrean with 1.8 KW Copper X-ray Tube). Raman spectroscopic analysis was performed using a Renishaw RM 1000B Micro-Raman Spectrometer with Ar - $514 \mathrm{~nm}$ excitation unit. The electrochemical studies of the electrodes and supercapacitor device were examined using an electrochemical workstation (Bio-Logic Science Instruments, model SP-150). The mass of CF substrate before and after the deposition of the active materials were measured using a microbalance (Mettler Toledo NewClassic MF, model MS 104S/03) to estimate the mass of active materials used in the supercapacitor electrodes.

\subsection{Kelvin probe force microscopy (KPFM) procedure}

The surface topography and the surface potential of the electrode materials were studied by atomic force microscopy (AFM) and KPFM (NanoIR2 AFM Bruker using PR-EX-KPFM cantilevers). KPFM measurements were performed using a two-pass method. During the first pass, the cantilever was mechanically driven at its resonant frequency to determine the sample's topography. During the second-pass scan, the cantilever was lifted by a fixed distance $(20 \mathrm{~nm})$ to maximize the effect of long-range forces and minimize the effect of short-range van der Waals forces. A bias which includes a DC and an AC component was applied to the cantilever to obtain a potential difference $\Delta \mathrm{V}=\mathrm{V}_{\mathrm{DC}}+\mathrm{V}_{\mathrm{AC}}$ between the tip and the sample. The $\mathrm{AC}$ component was used to electrically vibrate the cantilever at its resonant frequency while $\mathrm{V}_{\mathrm{DC}}$ was adjusted by the AFM controller to null the force gradient resulting from the differences between the tip work function ( $\left.\phi_{\text {tip }}\right)$ and the sample work function ( $\left.\phi_{\text {sample }}\right) . \mathrm{V}_{\mathrm{DC}}=\mathrm{V}_{\mathrm{CPD}}$ is recorded and used to obtain the KPFM maps. The work function of the sample, $\phi_{\text {sample }}$ is determined using the relationship $\phi_{\text {sample }}=\phi_{\text {tip }}$ $-\mathrm{e} \mathrm{V}_{\mathrm{CPD}}$, where $\mathrm{e}$ is the electronic charge and $\mathrm{V}_{\mathrm{CPD}}$ is contact potential difference between tip and the sample.

Before every measurement, the KPFM probe was calibrated to determine $\phi_{\text {tip. }}$. The calibration process involves the KPFM measurement of a freshly-exfoliated HOPG flake and evaluating the work function of HOPG using $\phi_{\mathrm{HOPG}}=\phi_{\text {tip }}-\mathrm{e} \mathrm{V}_{\mathrm{CPD}}$, where $\phi_{\mathrm{HOPG}}$ is $4.6+/-0.1$ $\mathrm{eV}$. The $\mathrm{V}_{\mathrm{CPD}}$ was extracted by averaging the values of the KPFM map on raw datasets. Thus, the $\mathrm{V}_{\mathrm{CPD}}$ value and the standard $\phi_{\mathrm{HOPG}}$ will help in determining the tip's work function. Followed by which, the electrode's work function is determined. For each electrode, at least 3 sets of samples were characterized and, on each sample, at least 3 positions were selected to confirm the repeatability of the measurements. All the measurements were done in ambient conditions. 


\subsection{Electrochemical Characterization}

All the electrodes were characterized by $\mathrm{CV}$ GCD and EIS measurement in a threeelectrode cell configuration. The active material $\left(\mathrm{NaMnO}_{2}\right.$ and $\left.\mathrm{MoO}_{2}\right)$ grown on $\mathrm{CF}$ was used as the working electrode, platinum foil as the counter electrode and $\mathrm{Ag} / \mathrm{AgCl}$ (in $1 \mathrm{M} \mathrm{KCl}$ ) as the reference electrode. An aqueous $1 \mathrm{M} \mathrm{Na}_{2} \mathrm{SO}_{4}$ solution was used as the electrolyte. An ASC device was fabricated with $\mathrm{NaMnO}_{2} / / \mathrm{MoO}_{2}$ after charge balance of the electrodes and all the electrochemical tests of the ASC were performed from 0 to $2.50 \mathrm{~V}$ in $1 \mathrm{M} \mathrm{Na}_{2} \mathrm{SO}_{4}$ electrolyte. The electrodes, $\mathrm{LiMnO}_{2}$, and $\mathrm{KMnO}_{2}$ were tested in three-electrode configurations in $1 \mathrm{M} \mathrm{Li}_{2} \mathrm{SO}_{4}$ and $1 \mathrm{M} \mathrm{K}_{2} \mathrm{SO}_{4}$ solution. The ASC device fabricated with $\mathrm{LiMnO}_{2} / / \mathrm{MoO}_{2}$ and $\mathrm{KMnO}_{2} / / \mathrm{MoO}_{2}$ was tested in $1 \mathrm{M} \mathrm{Li}_{2} \mathrm{SO}_{4}$ and $1 \mathrm{M} \mathrm{K}_{2} \mathrm{SO}_{4}$ solution, respectively, for the evolution studies.

\subsection{Charge balance between positive and negative electrode}

Achieving maximum device capacitance and longer cycle life is possible by maintaining a charge balance between the positive and negative electrode. The charge balance between the electrodes was performed to fabricate ASC using the following equation,

$$
\begin{aligned}
q^{+} & =q^{-} \\
M^{+} \times C^{+} \times \Delta \mathrm{E}^{+} & =M^{-} \times C^{-} \times \Delta \mathrm{E}^{-}
\end{aligned}
$$

Where $q^{+}$and $q^{-}$denote the charges stored on the positive and negative electrodes, respectively. The signs $M, C$, and $\Delta E$ represent the active electrode mass, specific capacitance, and the stable potential window of the respective electrodes. The mass of the active material is adjusted for charge balance as the three-electrode studies displayed a difference in both the electrode's specific capacitance and potential window.

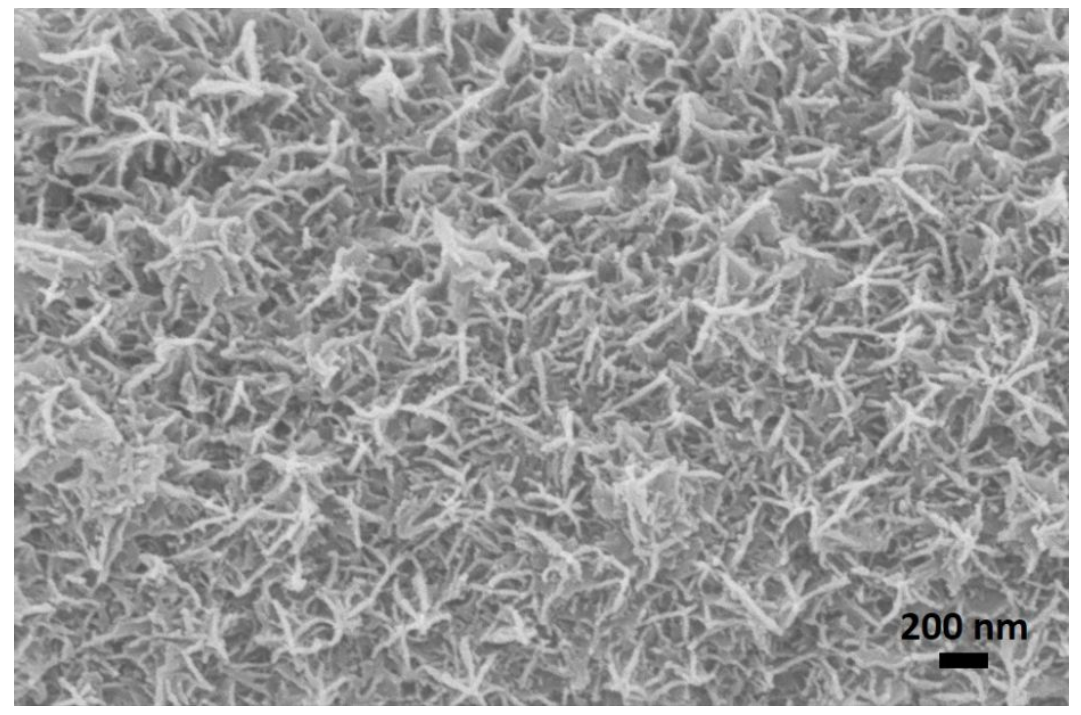

Figure S1. SEM image of $\mathrm{Mn}_{3} \mathrm{O}_{4}$ electrode 

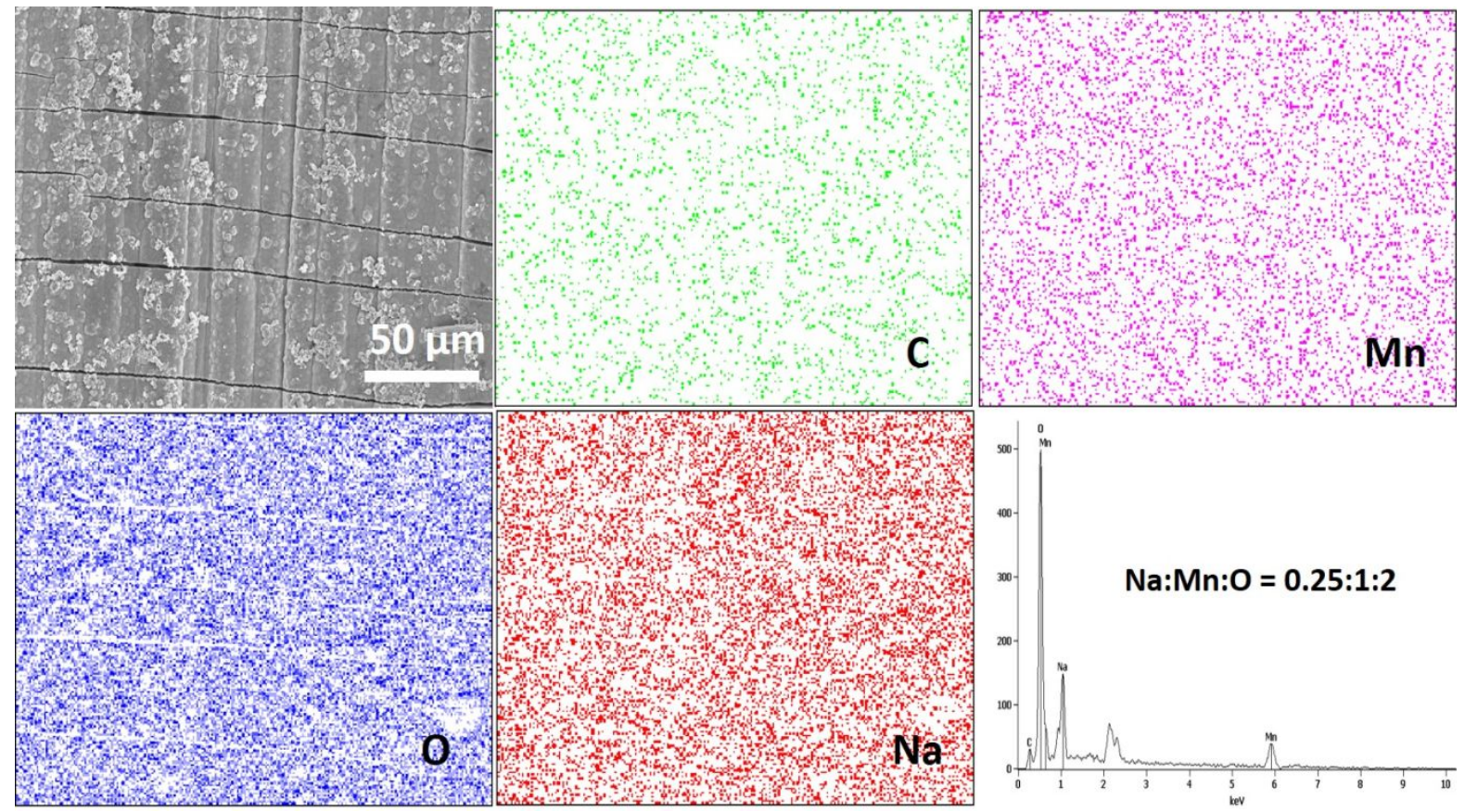

Figure S2. SEM-EDAX characterization of $\mathrm{NaMnO}_{2}$ electrode showing the presence of elements $\mathrm{C}, \mathrm{Mn}, \mathrm{O}$ and Na. 

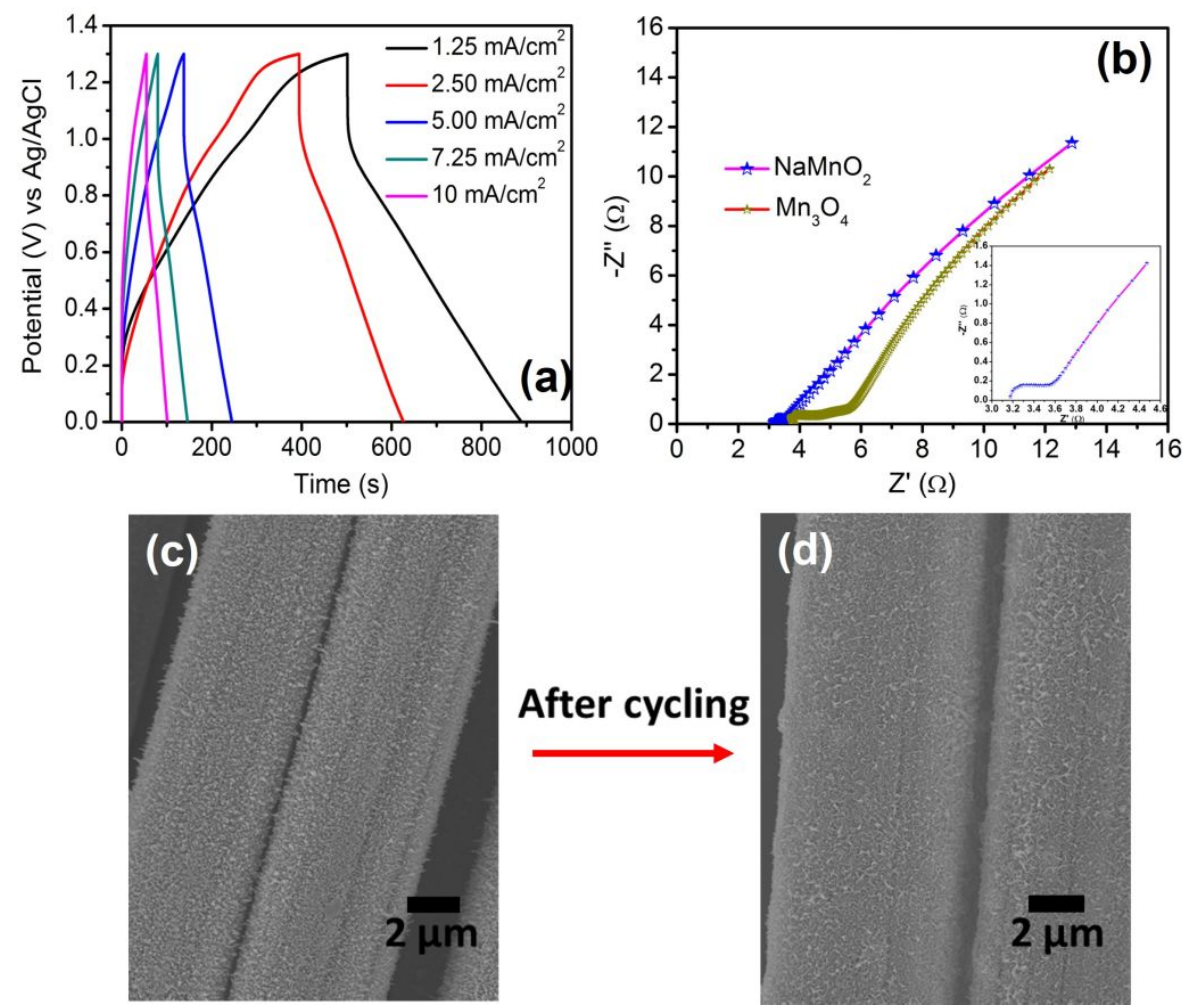

Figure S3. (a) GCD curves of $\mathrm{NaMnO}_{2}$ electrode, (b) Nyquist plot of $\mathrm{NaMnO}_{2}$ electrode vs $\mathrm{Mn}_{3} \mathrm{O}_{4}$ electrode with inset showing magnified portion of high-frequency region of $\mathrm{NaMnO}_{2}$ electrode, $\mathrm{SEM}$ image of (c) pristine $\mathrm{NaMnO}_{2}$ electrode and (d) cycled $\mathrm{NaMnO}_{2}$ electrode. 

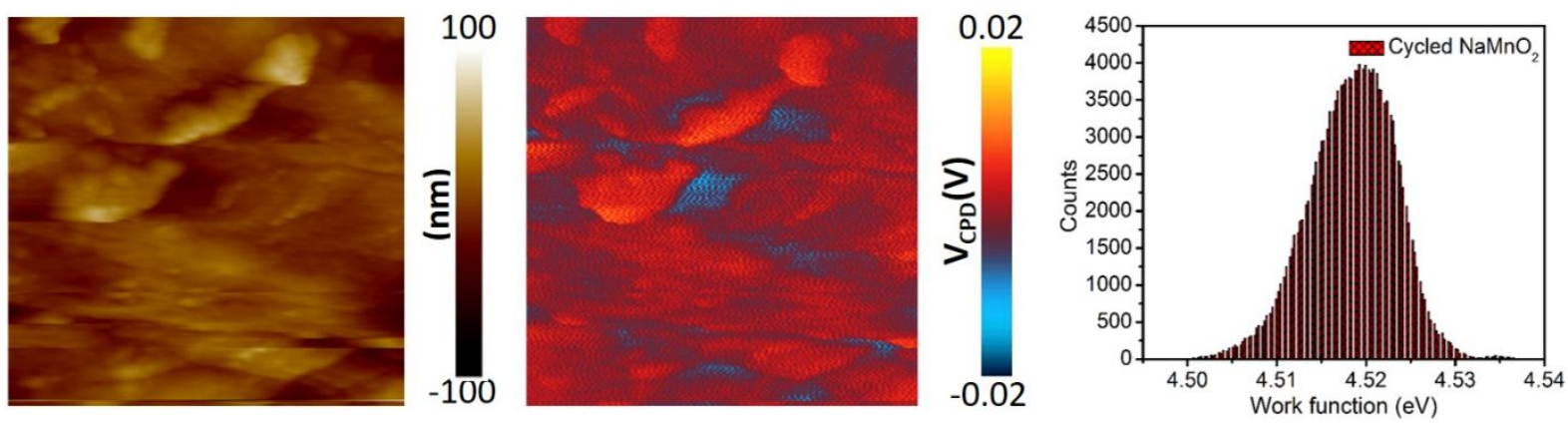

Figure S4. KPFM measurement of the work function of cycled $\mathrm{NaMnO}_{2}$ electrode 

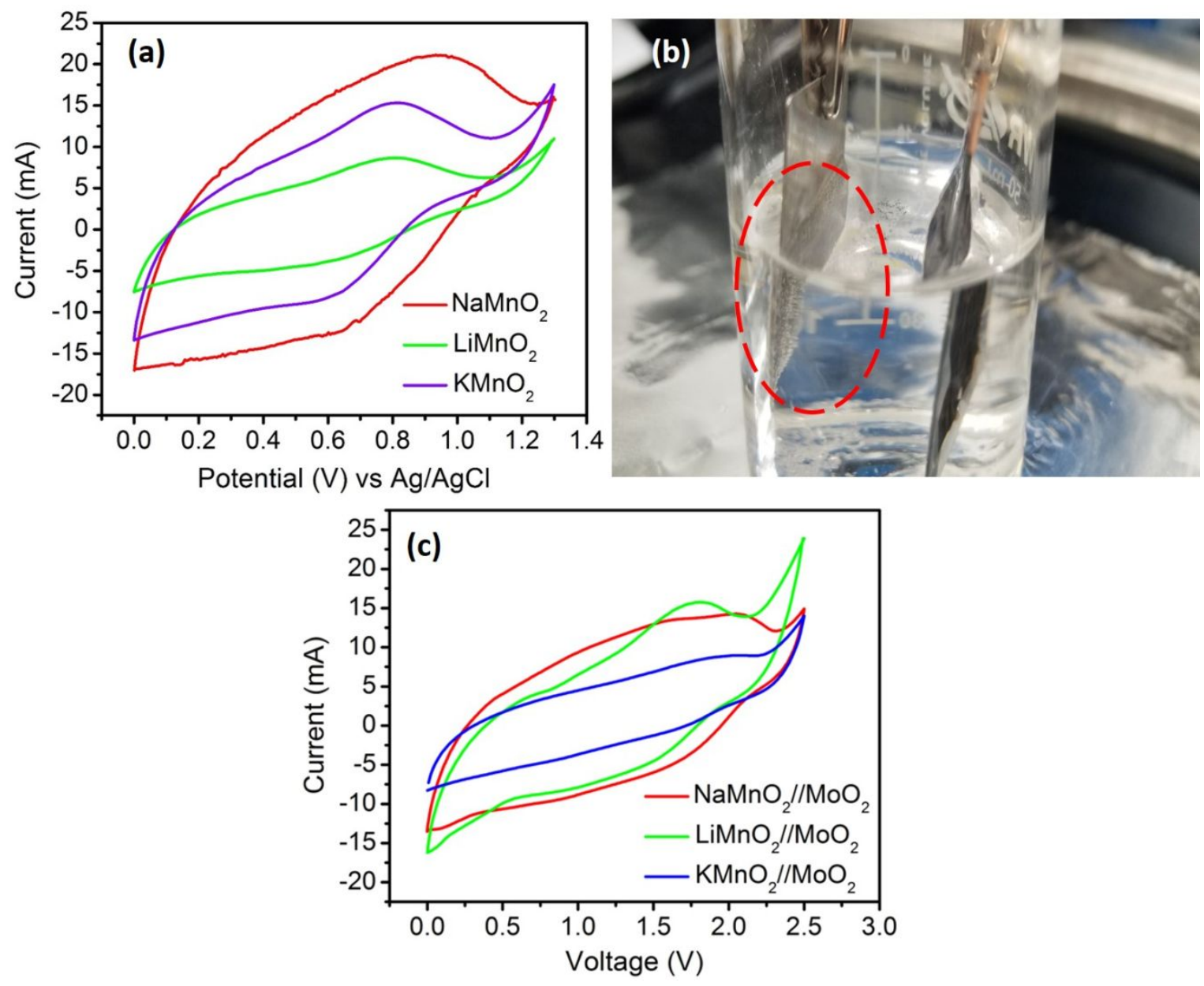

Figure S5. Oxygen evolution studies of various electrodes prepared (a) three electrode test of all the electrodes at a scan rate of $10 \mathrm{mV} \mathrm{s}^{-1}$ (b) Demonstration of the oxygen evolution at a voltage of $1.1 \mathrm{~V}$ for $\mathrm{KMnO}_{2}$ and $\mathrm{LiMnO}_{2}$ electrode (c) $\mathrm{CV}$ of various ASC devices at a scan rate of $10 \mathrm{mV} \mathrm{s}$ (showing the stable voltage window of $\mathrm{NaMnO}_{2} / / \mathrm{MoO}_{2}$ at higher voltage). 

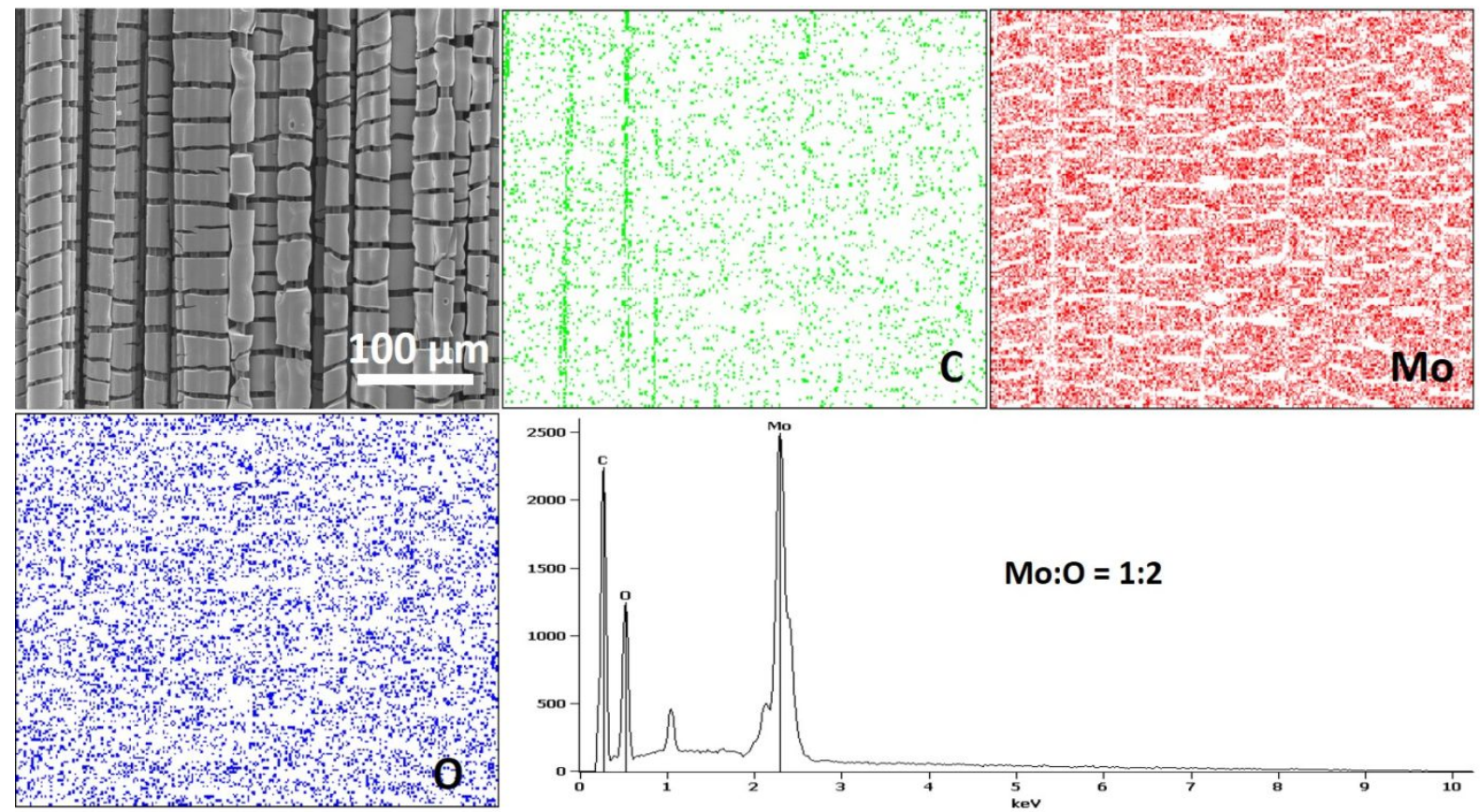

Figure S6. SEM-EDAX characterization of $\mathrm{MoO}_{2}$ electrode showing the presence of elements $\mathrm{C}$, Mo, and $\mathrm{O}$ 

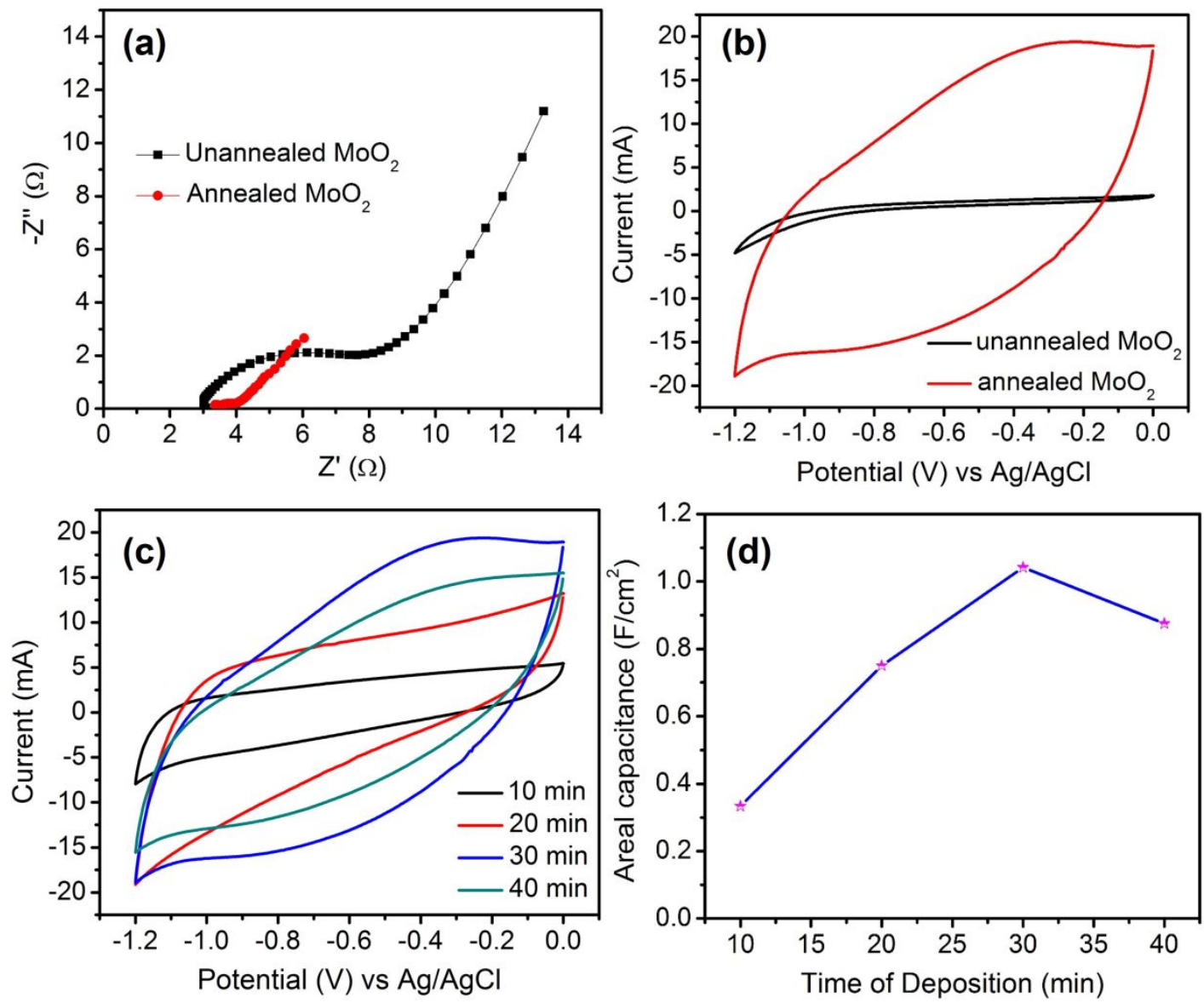

Figure S7. (a) Nyquist plot comparison of unannealed vs annealed $\mathrm{MoO}_{2}$ (b) $\mathrm{CV}$ profile comparison of unannealed vs annealed $\mathrm{MoO}_{2}$ at a scan rate of $5 \mathrm{mV} \mathrm{s}^{-1}$ (c) $\mathrm{CV}$ profile recorded at a scan rate of $5 \mathrm{mV} \mathrm{s}^{-1}$ of $\mathrm{MoO}_{2}$ electrodes prepared at different deposition time (d) Plot showing the time of deposition vs areal capacitance of $\mathrm{MoO}_{2}$ electrode at a scan rate of $5 \mathrm{mV} \mathrm{s} \mathrm{s}^{-1}$. 

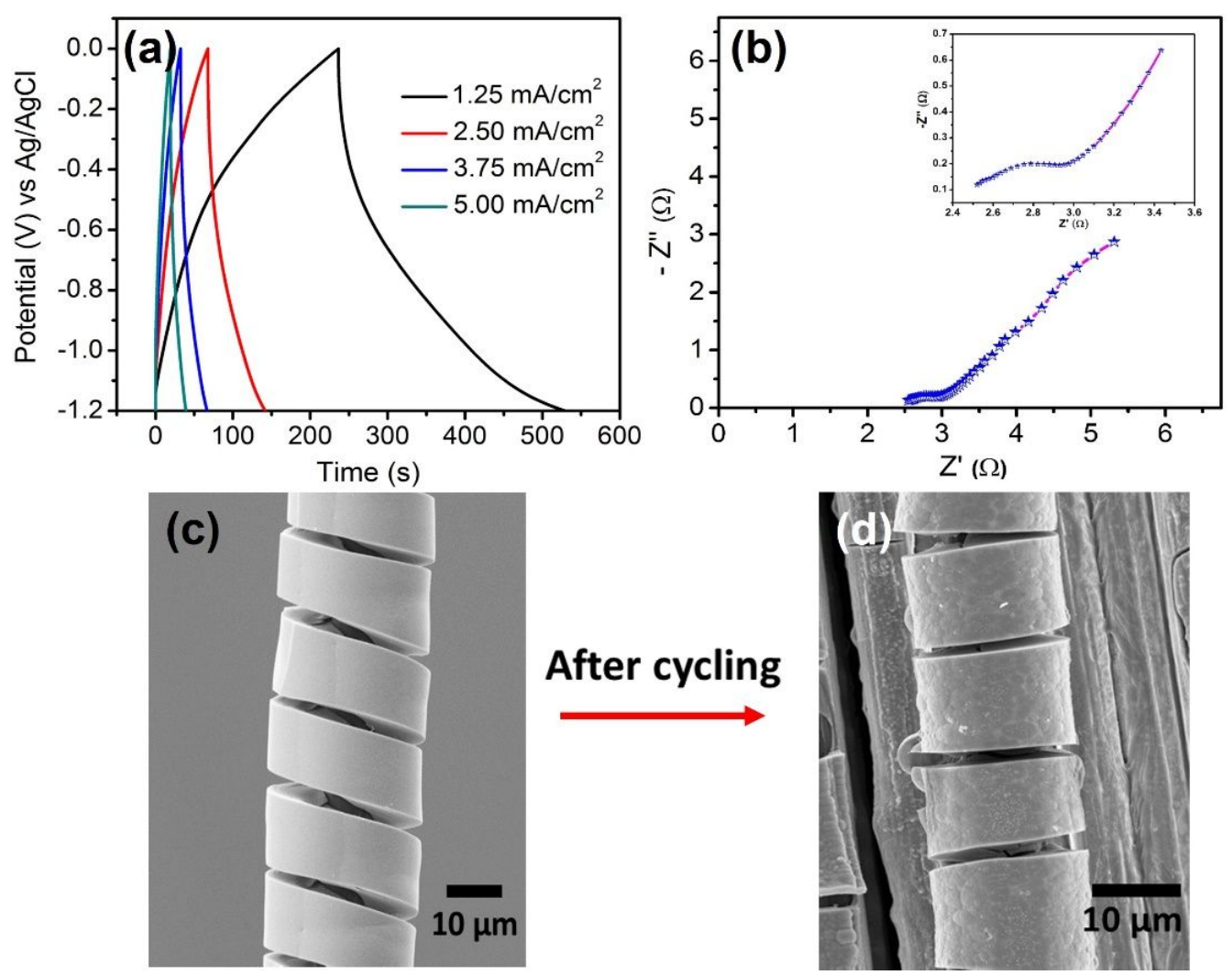

After cycling

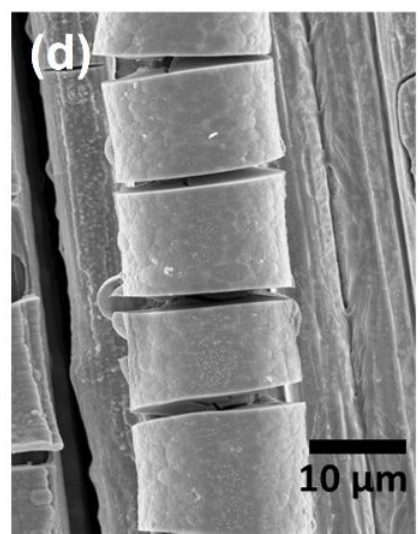

Figure S8. (a) GCD curves of $\mathrm{MoO}_{2}$ electrode, (b) Nyquist plot of $\mathrm{MoO}_{2}$ electrode with inset showing magnified portion of high-frequency region, SEM image of (c) pristine $\mathrm{MoO}_{2}$ electrode and (d) cycled $\mathrm{MoO}_{2}$ electrode. 

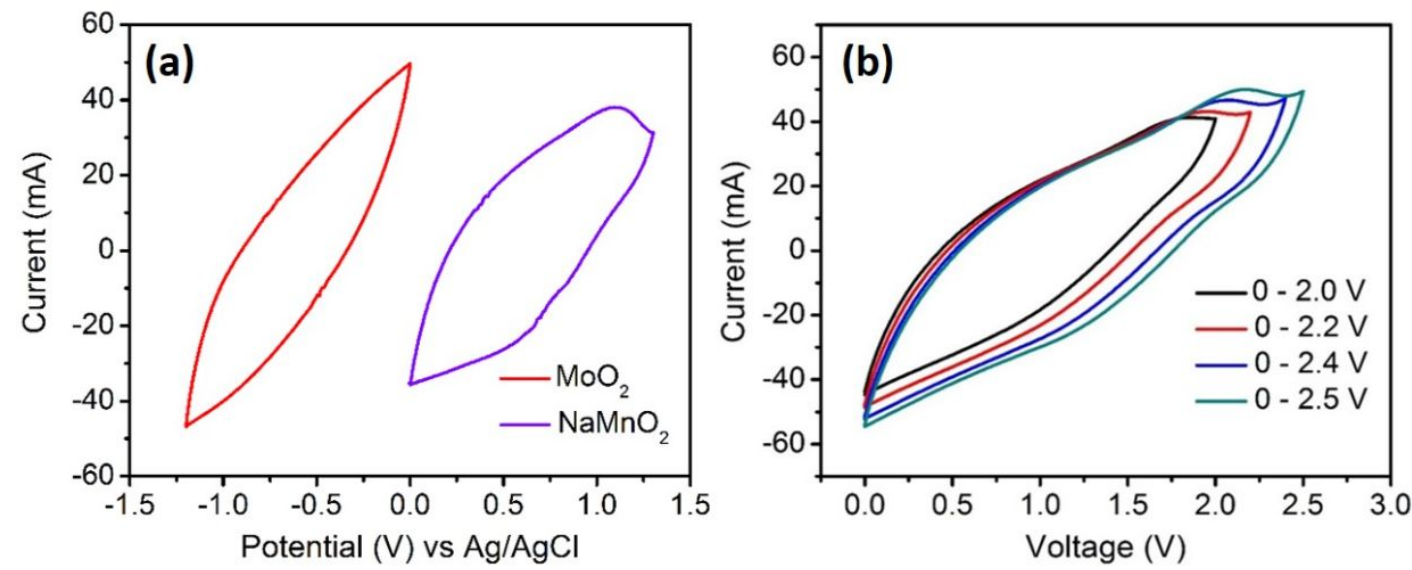

Figure S9. CV curves of the $\mathrm{MoO}_{2}$ and $\mathrm{NaMnO}_{2}$ electrode in a three-electrode system at a scan rate of $25 \mathrm{mV} \mathrm{s}^{-1}$ (b) $\mathrm{CV}$ curves of the $\mathrm{NaMnO}_{2} / / \mathrm{MoO}_{2}$ asymmetric device measured at different voltage windows at a scan rate of $100 \mathrm{mV} \mathrm{s}$. 
Table S1. Electrochemical performance comparison table of $\mathrm{NaMnO}_{2}$ electrode

\begin{tabular}{|c|c|c|c|c|c|c|c|}
\hline $\begin{array}{l}\text { S. } \\
\text { No }\end{array}$ & Electrode & Electrolyte & $\begin{array}{l}\text { Potential } \\
\text { range } \\
\text { (V) }\end{array}$ & $\begin{array}{l}\text { Areal } \\
\text { Capacitance } \\
\left(\mathrm{mF} \mathbf{c m}^{-2}\right)\end{array}$ & $\begin{array}{l}\text { Gravimetric } \\
\text { Capacitance } \\
\left(\mathrm{F} \mathrm{g}^{-1)}\right.\end{array}$ & $\begin{array}{l}\text { Cycling } \\
\text { stability }\end{array}$ & Ref \\
\hline 1. & $\begin{array}{l}\text { Carbon } \\
\text { nano } \\
\text { fiber/ } \\
\mathrm{MnO}_{2}\end{array}$ & $\begin{array}{l}1 \mathrm{M} \\
\mathrm{Na}_{2} \mathrm{SO}_{4}\end{array}$ & 0 to 0.8 & 525 & 110 & - & 2 \\
\hline 2. & $\begin{array}{l}\text { Activated } \\
\text { carbon@ } @ \delta \\
-\mathrm{MnO}_{2}\end{array}$ & $\begin{array}{l}1 \mathrm{M} \\
\mathrm{Na}_{2} \mathrm{SO}_{4}\end{array}$ & 0 to 1.0 & - & 345.1 & $\begin{array}{l}92.8 \% \\
\text { after } \\
5000 \\
\text { cycles }\end{array}$ & 3 \\
\hline 3. & $\begin{array}{l}\mathrm{MnO}_{2} / \\
\mathrm{MoS}_{2}\end{array}$ & $\begin{array}{l}1 \mathrm{M} \\
\mathrm{Na}_{2} \mathrm{SO}_{4}\end{array}$ & 0 to 1.0 & 224 & - & $\begin{array}{l}90 \% \\
\text { after } \\
3000 \\
\text { cycles }\end{array}$ & 4 \\
\hline 4. & $\begin{array}{l}\mathrm{MnO}_{2} @ \\
\text { Carbon }\end{array}$ & $\begin{array}{l}1 \mathrm{M} \\
\mathrm{Na}_{2} \mathrm{SO}_{4}\end{array}$ & 0 to 1.0 & - & 30.5 & - & 5 \\
\hline 5. & $\begin{array}{l}\mathrm{Mn}_{2} \mathrm{O}_{3} @ \\
\mathrm{MnO}_{2}-0.3\end{array}$ & $\begin{array}{l}1 \mathrm{M} \\
\mathrm{Na}_{2} \mathrm{SO}_{4}\end{array}$ & 0 to 1.0 & - & 225 & $\begin{array}{l}86.5 \% \\
\text { after } \\
5000 \\
\text { cycles }\end{array}$ & 6 \\
\hline 6. & $\begin{array}{l}\text { Porous } \\
\text { Graphene } \\
\text { (a) } \mathrm{Mn}_{3} \mathrm{O}_{4}\end{array}$ & $\begin{array}{l}1 \mathrm{M} \\
\mathrm{Na}_{2} \mathrm{SO}_{4}\end{array}$ & $\begin{array}{l}-0.2 \text { to } \\
0.8\end{array}$ & - & 208.3 & $\begin{array}{l}86 \% \\
\text { after } \\
2000 \\
\text { cycles }\end{array}$ & 7 \\
\hline 7. & $\mathrm{MnO}_{2}$ & $\begin{array}{l}1 \mathrm{M} \\
\mathrm{Na}_{2} \mathrm{SO}_{4}\end{array}$ & 0 to 1.0 & - & 291 & $\begin{array}{l}90.9 \% \\
\text { after } \\
5000 \\
\text { cycles }\end{array}$ & 8 \\
\hline 8. & $\begin{array}{l}\mathrm{N} / \mathrm{P}- \\
\text { Hollow } \\
\text { carbon @ } \\
\mathrm{MnO}_{2}\end{array}$ & $\begin{array}{l}1 \mathrm{M} \\
\mathrm{Na}_{2} \mathrm{SO}_{4}\end{array}$ & $\begin{array}{l}-0.1 \text { to } \\
0.9\end{array}$ & - & 222 & $\begin{array}{l}92 \% \\
\text { after } \\
5000 \\
\text { cycles }\end{array}$ & 9 \\
\hline 9. & $\begin{array}{l}\text { Graphene } \\
\text { @ } \mathrm{MnO}_{2}\end{array}$ & $\begin{array}{l}1 \mathrm{M} \\
\mathrm{Na}_{2} \mathrm{SO}_{4}\end{array}$ & 0 to 1.0 & - & 245 & $\begin{array}{l}92.1 \% \\
\text { after } \\
10000 \\
\text { cycles }\end{array}$ & 10 \\
\hline 10. & $\mathrm{NaMnO}_{2}$ & $\begin{array}{l}1 \mathrm{M} \\
\mathrm{Na}_{2} \mathrm{SO}_{4}\end{array}$ & 0 to 1.3 & 1000 & 328 & $\begin{array}{l}\sim 97.7 \% \\
\text { after } \\
5000 \\
\text { cycles }\end{array}$ & $\begin{array}{l}\text { Our } \\
\text { work }\end{array}$ \\
\hline
\end{tabular}


Table S2. Electrochemical performance comparison table of $\mathrm{MoO}_{2}$ electrode

\begin{tabular}{|c|c|c|c|c|c|c|c|}
\hline $\begin{array}{l}\text { S. } \\
\text { No }\end{array}$ & Electrode & Electrolyte & $\begin{array}{l}\text { Potential } \\
\text { range } \\
\text { (V) }\end{array}$ & $\begin{array}{l}\text { Areal } \\
\text { Capacitance } \\
\left(\mathrm{mF} \mathrm{cm}^{-2}\right)\end{array}$ & $\begin{array}{l}\text { Gravimetric } \\
\text { Capacitance } \\
\left(\mathrm{F} \mathrm{g} \mathrm{g}^{-1}\right)\end{array}$ & $\begin{array}{l}\text { Cycling } \\
\text { stability }\end{array}$ & Ref \\
\hline 1. & $\begin{array}{l}\mathrm{WO}_{3-\mathrm{x}} / \\
\mathrm{MoO}_{3-\mathrm{x}}\end{array}$ & $\begin{array}{l}0.5 \mathrm{M} \\
\mathrm{Na}_{2} \mathrm{SO}_{4}\end{array}$ & 0 to -1.1 & 500 & - & - & 11 \\
\hline 2. & $\begin{array}{l}\mathrm{MoO}_{3} / \mathrm{GO} \\
\text { /MWCNT } \\
\text { /Graphite }\end{array}$ & $1 \mathrm{M} \mathrm{KCl}$ & $\begin{array}{l}-0.2 \text { to } \\
1.0\end{array}$ & 115 & - & - & 12 \\
\hline 3. & $\begin{array}{l}\text { Carbon } \\
\text { fiber/ } \\
\mathrm{MoO}_{3}\end{array}$ & $1 \mathrm{M} \mathrm{KOH}$ & 0 to -1.0 & 27.5 & - & - & 13 \\
\hline 4. & $\begin{array}{l}\mathrm{Ag} \text { QDs/ } \\
\mathrm{MoO}_{3}\end{array}$ & $\begin{array}{l}1 \mathrm{M} \\
\mathrm{Na}_{2} \mathrm{SO}_{4}\end{array}$ & 0 to -1.0 & 735.6 & - & $\begin{array}{l}108.8 \% \\
\text { after } \\
50000 \\
\text { cycles }\end{array}$ & 14 \\
\hline 5. & CS- $\mathrm{MoO}_{2}$ & $6 \mathrm{M} \mathrm{KOH}$ & $\begin{array}{l}-0.2 \text { to }- \\
1.0\end{array}$ & - & 245 & $\begin{array}{l}100 \% \\
\text { after } \\
5000 \\
\text { cycles }\end{array}$ & 15 \\
\hline 6. & $\begin{array}{l}\mathrm{MoS}_{2} / \\
\mathrm{MoO}_{\mathrm{x}} / \\
\text { Activated } \\
\text { carbon }\end{array}$ & $1 \mathrm{M} \mathrm{H}_{2} \mathrm{SO}_{4}$ & $\begin{array}{l}-0.8 \text { to } \\
+0.8\end{array}$ & - & 230 & $\begin{array}{l}128 \% \\
\text { after } \\
1500 \\
\text { cycles }\end{array}$ & 16 \\
\hline 7. & $\begin{array}{l}\mathrm{MoS}_{2} / \\
\mathrm{MoO}_{2} @ \\
\mathrm{CNT}\end{array}$ & $6 \mathrm{M} \mathrm{KOH}$ & 0 to 0.55 & - & 228.4 & $\begin{array}{l}187.1 \% \\
\text { after } \\
25000 \\
\text { cycles }\end{array}$ & 17 \\
\hline 8. & $\begin{array}{l}\mathrm{MoO}_{2} @ \mathrm{~N} \\
\mathrm{CT}\end{array}$ & $\begin{array}{l}1 \mathrm{M} \\
\mathrm{Na}_{2} \mathrm{SO}_{4}\end{array}$ & 0 to -1.0 & - & 254.8 & $\begin{array}{l}96.3 \% \\
\text { after } \\
10000 \\
\text { cycles }\end{array}$ & 18 \\
\hline 9. & $\mathrm{MoO}_{2}$ & $1 \mathrm{M} \mathrm{H}_{2} \mathrm{SO}_{4}$ & $\begin{array}{l}0.2 \text { to }- \\
0.35\end{array}$ & - & 204.7 & $\begin{array}{l}90.6 \% \\
\text { after } \\
1000 \\
\text { cycles }\end{array}$ & 19 \\
\hline 10. & $\mathrm{MoO}_{2}$ & $\begin{array}{l}1 \mathrm{M} \\
\mathrm{Na}_{2} \mathrm{SO}_{4}\end{array}$ & 0 to -1.2 & 1040 & 260 & $\begin{array}{l}\sim 96.5 \% \\
\text { after } \\
5000 \\
\text { cycles }\end{array}$ & $\begin{array}{l}\text { Our } \\
\text { work }\end{array}$ \\
\hline
\end{tabular}


Table S3. Stable voltage window of various ASC devices with respect to work function difference of the metal oxide electrodes used.

\begin{tabular}{|l|l|l|l|l|l|l|}
\hline S. No & $\begin{array}{l}\text { Positive } \\
\text { electrode }\end{array}$ & $\begin{array}{l}\text { Average } \\
\text { Work } \\
\text { function } \\
(\mathbf{e V})\end{array}$ & $\begin{array}{l}\text { Negative } \\
\text { electrode }\end{array}$ & $\begin{array}{l}\text { Average } \\
\text { Work } \\
\text { function } \\
\mathbf{e V}\end{array}$ & $\begin{array}{l}\text { Average Work } \\
\text { function } \\
\text { difference (eV) }\end{array}$ & $\begin{array}{l}\text { Stable Output } \\
\text { Voltage (V) }\end{array}$ \\
\hline 1. & $\mathrm{NaMnO}_{2}$ & 4.50 & $\mathrm{MoO}_{2}$ & 6.50 & 2.00 & 2.50 \\
\hline 2. & $\mathrm{LiMnO}_{2}$ & 4.72 & $\mathrm{MoO}_{2}$ & 6.50 & 1.78 & 2.25 \\
\hline 3. & $\mathrm{KMnO}_{2}$ & 4.78 & $\mathrm{MoO}_{2}$ & 6.50 & 1.72 & 2.20 \\
\hline
\end{tabular}

Table S4. Maximum electrochemical performance evaluation of various ASC devices

\begin{tabular}{|c|c|c|c|c|c|}
\hline S. No & ASC device & Electrolyte & 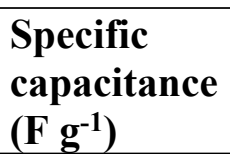 & $\begin{array}{l}\text { Energy Density } \\
\left(W \mathrm{~h} \mathrm{~kg}^{-1}\right)\end{array}$ & $\begin{array}{l}\text { Power Density } \\
\left(\mathrm{W} \mathrm{kg}^{-1}\right)\end{array}$ \\
\hline 1. & $\mathrm{NaMnO}_{2} / / \mathrm{MoO}_{2}$ & $1 \mathrm{M} \mathrm{Na}_{2} \mathrm{SO}_{4}$ & 89.6 & 78 & 565 \\
\hline 2. & $\mathrm{LiMnO}_{2} / / \mathrm{MoO}_{2}$ & $1 \mathrm{M} \mathrm{Li}_{2} \mathrm{SO}_{4}$ & 61.4 & 53.3 & 390 \\
\hline 3. & $\mathrm{KMnO}_{2} / / \mathrm{MoO}_{2}$ & $1 \mathrm{M} \mathrm{K}_{2} \mathrm{SO}_{4}$ & 57.6 & 50 & 365 \\
\hline
\end{tabular}


Table S5. Electrochemical performance comparison table of $\mathrm{NaMnO}_{2} / / \mathrm{MoO}_{2} \mathrm{ASC}$

\begin{tabular}{|c|c|c|c|c|c|c|c|c|c|}
\hline $\begin{array}{l}\text { S. } \\
\text { No }\end{array}$ & $\begin{array}{l}\text { ASC } \\
\text { System }\end{array}$ & $\begin{array}{l}\text { Electroly } \\
\text { te }\end{array}$ & $\begin{array}{l}\text { Voltage } \\
\text { (V) }\end{array}$ & $\begin{array}{l}\text { Areal } \\
\text { Capa } \\
\text { citanc } \\
\text { e }(\mathbf{m F} \\
\left.\mathbf{c m}^{-2}\right)\end{array}$ & $\begin{array}{l}\text { Gravim } \\
\text { etric } \\
\text { Capacit } \\
\text { ance }(F \\
\left.\mathbf{g}^{-1}\right)\end{array}$ & $\begin{array}{l}\text { Cyclin } \\
\text { g } \\
\text { stabilit } \\
\text { y }\end{array}$ & $\begin{array}{l}\text { Energy } \\
\text { Density } \\
(\mathbf{W ~ h} \\
\left.\mathbf{k g}^{-1}\right)\end{array}$ & $\begin{array}{l}\text { Power } \\
\text { Density } \\
(W \\
\left.\mathbf{k g}^{-1}\right)\end{array}$ & Ref \\
\hline 1. & $\begin{array}{l}\mathrm{CNF} / \mathrm{MnO}_{2} \\
/ / \\
\mathrm{CNF} / \mathrm{Bi}_{2} \mathrm{O}_{3}\end{array}$ & $\begin{array}{l}1 \mathrm{M} \\
\mathrm{Na}_{2} \mathrm{SO}_{4}\end{array}$ & 1.8 & 97 & 25.2 & $\begin{array}{l}85 \% \\
\text { after } \\
4000 \\
\text { cycles }\end{array}$ & 11.3 & 352.6 & 2 \\
\hline 2. & $\begin{array}{l}\mathrm{MnO}_{2} @ \mathrm{C} \\
\mathrm{NT} / / \\
\mathrm{MoO}_{3} @ \mathrm{C} \\
\mathrm{NT}\end{array}$ & $\begin{array}{l}1 \mathrm{M} \\
\mathrm{Na}_{2} \mathrm{SO}_{4}\end{array}$ & 2 & - & 61 & $\begin{array}{l}\sim 96.8 \\
\% \text { after } \\
10000 \\
\text { cycles }\end{array}$ & 27.8 & 524 & 20 \\
\hline 3. & $\begin{array}{l}\mathrm{Mn}_{2} \mathrm{O}_{3} @ \mathrm{M} \\
\mathrm{nO}_{2^{-}}-0.3 \\
/ / \mathrm{AC}\end{array}$ & $\begin{array}{l}1 \mathrm{M} \\
\mathrm{Na}_{2} \mathrm{SO}_{4}\end{array}$ & 1.8 & - & 60 & $\begin{array}{l}84 \% \\
\text { after } \\
5000 \\
\text { cycles }\end{array}$ & 27 & 90 & 6 \\
\hline 4. & $\begin{array}{l}\mathrm{MnO}_{2} / / \\
(\mathrm{Fe}, \mathrm{Cr})_{2} \mathrm{O}_{3}\end{array}$ & $\begin{array}{l}1 \mathrm{M} \\
\mathrm{Na}_{2} \mathrm{SO}_{4}\end{array}$ & 2.0 & 4.3 & 37 & $\begin{array}{l}115 \% \\
\text { after } \\
5000 \\
\text { cycles }\end{array}$ & 20.89 & 2174 & 21 \\
\hline 5. & $\begin{array}{l}\mathrm{N} / \mathrm{P}- \\
\text { carbon } \\
@ \text { MnO } \\
\text { // N/P- } \\
\text { carbon }\end{array}$ & $\begin{array}{l}1 \mathrm{M} \\
\mathrm{Na}_{2} \mathrm{SO}_{4}\end{array}$ & 1.8 & - & 71.58 & $\begin{array}{l}94.5 \% \\
\text { after } \\
3000 \\
\text { cycles }\end{array}$ & 32.21 & 449.8 & 9 \\
\hline 6. & $\begin{array}{l}\mathrm{MoO}_{2} / \mathrm{AR} \\
\mathrm{CF} / / \\
\mathrm{MnO}_{2} / \mathrm{AR} \\
\mathrm{CF}\end{array}$ & $\begin{array}{l}1 \mathrm{M} \\
\mathrm{Na}_{2} \mathrm{SO}_{4}\end{array}$ & 2.2 & - & 58.6 & $\begin{array}{l}93.75 \\
\% \text { after } \\
3000 \\
\text { cycles }\end{array}$ & 39.4 & - & 22 \\
\hline 7. & $\begin{array}{l}\mathrm{GrMnO}_{2} / / \\
\mathrm{GrMoO}_{3}\end{array}$ & $\begin{array}{l}1 \mathrm{M} \\
\mathrm{Na}_{2} \mathrm{SO}_{4}\end{array}$ & 2.0 & - & 307 & $\begin{array}{l}100 \% \\
\text { after } \\
1000 \\
\text { cycles }\end{array}$ & 42.6 & 276 & 23 \\
\hline 8. & $\begin{array}{l}\mathrm{MnO} @ \mathrm{NC} \\
\mathrm{T} / / \\
\mathrm{MoO}_{2} @ \mathrm{~N} \\
\mathrm{CT}\end{array}$ & $\begin{array}{l}1 \mathrm{M} \\
\mathrm{Na}_{2} \mathrm{SO}_{4}\end{array}$ & 1.8 & - & 99.6 & $\begin{array}{l}92.5 \% \\
\text { after } \\
1000 \\
\text { cycles }\end{array}$ & 44.82 & 900 & 18 \\
\hline 9. & $\begin{array}{l}\mathrm{Ag} \text { QDs/ } \\
\mathrm{MnO}_{2} / / \mathrm{Ag} \\
\mathrm{QDs} / \\
\mathrm{MoO}_{3}\end{array}$ & $\begin{array}{l}\mathrm{Na}_{2} \mathrm{SO}_{4} / \\
\text { PVA }\end{array}$ & 2.0 & $\begin{array}{l}105.3 \\
5\end{array}$ & 119 & $\begin{array}{l}90 \% \\
\text { after } \\
5000 \\
\text { cycles }\end{array}$ & 51.89 & 22.6 & 14 \\
\hline 10. & $\begin{array}{l}\mathrm{NaMnO}_{2} / / \\
\mathrm{MoO}_{2}\end{array}$ & $\begin{array}{l}1 \mathrm{M} \\
\mathrm{Na}_{2} \mathrm{SO}_{4}\end{array}$ & 2.5 & 373 & 89.6 & $\begin{array}{l}98.6 \\
\% \text { after }\end{array}$ & 78 & 565 & $\begin{array}{l}\text { Our } \\
\text { work }\end{array}$ \\
\hline
\end{tabular}




\begin{tabular}{|l|l|l|l|l|l|l|l|l|}
\hline & & & & & $\begin{array}{l}5000 \\
\text { cycles }\end{array}$ & & & \\
\hline
\end{tabular}

\section{Reference}

1. Sambath Kumar, K.; Cherusseri, J.; Thomas, J., Two-Dimensional Mn3O4 Nanowalls Grown on Carbon Fibers as Electrodes for Flexible Supercapacitors. ACS omega 2019, 4 (2), 4472-4480.

2. Xu, H. H.; Hu, X. L.; Yang, H. L.; Sun, Y. M.; Hu, C. C.; Huang, Y. H., Flexible Asymmetric MicroSupercapacitors Based on $\mathrm{Bi} 2 \mathrm{O} 3$ and $\mathrm{MnO} 2$ Nanoflowers: Larger Areal Mass Promises Higher Energy Density. Adv Energy Mater 2015, 5 (6), 1401882.

3. Wang, X.; Chen, S.; Li, D. H.; Sun, S. L.; Peng, Z.; Komarneni, S.; Yang, D. J., Direct Interfacial Growth of $\mathrm{MnO} 2$ Nanostructure on Hierarchically Porous Carbon for High-Performance Asymmetric Supercapacitors. Acs Sustain Chem Eng 2018, 6 (1), 633-641.

4. $\quad$ Zhang, H. H.; Wei, J.; Yan, Y.; Guo, Q. J.; Xie, L. Q.; Yang, Z. C.; He, J.; Qi, W.; Cao, Z. S.; Zhao, X. H.; Pan, P.; Li, H. Y.; Zhang, K. L.; Zhao, J. S.; Li, X.; Zhang, P.; Shah, K. W., Facile and scalable fabrication of $\mathrm{MnO} 2$ nanocrystallines and enhanced electrochemical performance of $\mathrm{MnO} 2 / \mathrm{MoS} 2$ inner heterojunction structure for supercapacitor application. J Power Sources 2020, 450, 227616.

5. Li, M. L.; Yu, J.; Wang, X. D.; Yang, Z. L., 3D porous MnO2@carbon nanosheet synthesized from rambutan peel for high-performing supercapacitor electrodes materials. App/ Surf Sci 2020, 530, 147230. 6. Lu, W.; Li, Y.; Yang, M.; Jiang, X.; Zhang, Y. X.; Xing, Y., Construction of Hierarchical Mn2O3@MnO2 Core-Shell Nanofibers for Enhanced Performance Supercapacitor Electrodes. Acs Appl Energ Mater 2020, 3 (9), 8190-8197.

7. Wang, T.; Le, Q. J.; Guo, X. L.; Huang, M.; Liu, X. Y.; Dong, F.; Zhang, J. T.; Zhang, Y. X., Preparation of Porous Graphene@Mn3O4 and Its Application in the Oxygen Reduction Reaction and Supercapacitor. Acs Sustain Chem Eng 2019, 7 (1), 831-837.

8. Xu, K. B.; Li, S. J.; Yang, J. M.; Hu, J. Q., Hierarchical hollow MnO2 nanofibers with enhanced supercapacitor performance. J Colloid Interf Sci 2018, 513, 448-454.

9. Wu, P. C.; Gao, M.; Yu, S. C.; Feng, M. L.; Liu, S. H.; Fu, J. W., MnO2 nanosheets grown on N and P co-doped hollow carbon microspheres for high performance asymmetric supercapacitor. Electrochim Acta 2020, 354, 136681 .

10. Wang, L.; Ouyang, Y.; Jiao, X. Y.; Xia, X. F.; Lei, W.; Hao, Q. L., Polyaniline-assisted growth of MnO2 ultrathin nanosheets on graphene and porous graphene for asymmetric supercapacitor with enhanced energy density. Chem Eng J 2018, 334, 1-9.

11. Xiao, X.; Ding, T.; Yuan, L.; Shen, Y.; Zhong, Q.; Zhang, X.; Cao, Y.; Hu, B.; Zhai, T.; Gong, L., WO3$\mathrm{x} / \mathrm{MoO} 3-\mathrm{x}$ core/shell nanowires on carbon fabric as an anode for all-solid-state asymmetric supercapacitors. Adv Energy Mater 2012, 2 (11), 1328-1332.

12. Faraji, M.; Abedini, A., Fabrication of electrochemically interconnected MoO3/GO/MWCNTs/graphite sheets for high performance all-solid-state symmetric supercapacitor. Int J Hydrogen Energ 2019, 44 (5), 2741-2751.

13. Noh, J.; Yoon, C.-M.; Kim, Y. K.; Jang, J., High performance asymmetric supercapacitor twisted from carbon fiber/MnO2 and carbon fiber/MoO3. Carbon 2017, 116, 470-478.

14. Zhang, X.; Fu, Q.; Huang, H.; Wei, L.; Guo, X., Silver-quantum-dot-modified $\mathrm{MoO} 3$ and $\mathrm{MnO} 2$ paper-like freestanding films for flexible solid-state asymmetric supercapacitors. Small 2019, 15 (13), 1805235.

15. Liang, J. W.; Zhang, X. N.; Yan, C.; Wang, Y. X.; Norton, M. L.; Wei, X. J.; Donley, C.; Zhu, Y.; Xiao, P.; Zhang, Y. H., Preparation and enhanced supercapacitance performance of carbonized silk by feeding silkworms MoO 2 nanoparticles. Materials \& Design 2020, 196, 109137. 
16. Sari, F. N. I.; Ting, J. M., MoS2/MoOx-Nanostructure-Decorated Activated Carbon Cloth for Enhanced Supercapacitor Performance. Chemsuschem 2018, 11 (5), 897-906.

17. Tian, Y. R.; Du, H. S.; Zhang, M. M.; Zheng, Y. Y.; Guo, Q. P.; Zhang, H. P.; Luo, J. J.; Zhang, X. Y., Microwave synthesis of MoS2/MoO2@CNT nanocomposites with excellent cycling stability for supercapacitor electrodes. J Mater Chem C 2019, 7 (31), 9545-9555.

18. Yan, Y. T.; Lin, J. H.; Jiang, J.; Wang, H. H.; Qi, J. L.; Zhong, Z. X.; Cao, J.; Fei, W. D.; Feng, J. C., A general strategy to construct $\mathrm{N}$-doped carbon-confined $\mathrm{MoO} 2$ and $\mathrm{MnO}$ for high-performance hybrid supercapacitors. Vacuum 2019, 165, 179-185.

19. Ou, P.; Zhou, Q. Y.; Li, J.; Chen, W.; Huang, J. G.; Yang, L. Q.; Liao, J.; Sheng, M. Q., Facile ethylene glycol-assisted hydrothermal synthesis of $\mathrm{MoO} 2$ nanospheres for high-performance supercapacitors. Mater Res Express 2019, 6 (9), 095044.

20. Lee, T. H.; Pham, D. T.; Sahoo, R.; Seok, J.; Luu, T. H. T.; Lee, Y. H., High energy density and enhanced stability of asymmetric supercapacitors with mesoporous MnO2@ CNT and nanodot MoO3@ CNT free-standing films. Energy Storage Mater 2018, 12, 223-231.

21. Deshmukh, P. R.; Sohn, Y.; Shin, W. G., Electrochemical performance of facile developed aqueous asymmetric ( $\mathrm{Fe}, \mathrm{Cr})(2) \mathrm{O}-3 / / \mathrm{MnO} 2$ supercapacitor. Electrochim Acta 2018, 285, 381-392.

22. Zhao, C. J.; Hu, Y. Q.; Zhou, Y. N.; Li, N.; Ding, Y. W.; Guo, J. J.; Zhao, C. H.; Yang, Y. R., Aerobic Recovered Carbon Fiber Support-Based $\mathrm{MoO} 2 / / \mathrm{MnO} 2$ Asymmetric Supercapacitor with a Widened Voltage Window. Energ Fuel 2021, 35 (8), 6909-6920.

23. Chang, J.; Jin, M.; Yao, F.; Kim, T. H.; Le, V. T.; Yue, H.; Gunes, F.; Li, B.; Ghosh, A.; Xie, S., Asymmetric supercapacitors based on graphene/MnO2 nanospheres and graphene/MoO3 nanosheets with high energy density. Adv Funct Mater 2013, 23 (40), 5074-5083. 